\title{
Vulnerabilidad socioambiental en Monte Caseros, Corrientes: aportes al estudio de las problemáticas socio-espaciales de la localidad
}

Laura Zulaica ${ }^{1}$

Rosana Ferraro ${ }^{2}$

\section{Artículo científico}

Material original autorizado para su primera publicación en el Journal de Ciencias Sociales, Revista Académica de la Facultad de Ciencias Sociales de la Universidad de Palermo.

Recibido: 27-4-2016

Aceptado: $11-7-2016$

\section{Resumen}

Las ciudades se encuentran expuestas a diversos fenómenos naturales y problemas ambientales que constituyen un desafío para la gestión local, cuya incertidumbre es cada vez mayor en un contexto de cambio climático. En la localidad de Monte Caseros, Corrientes, las crecientes del río Uruguay se traducen en eventos de inundaciones, como los evidenciados hacia fines de 2015 y principios de 2016. Estos fenómenos pueden adquirir mayor o menor relevancia de acuerdo con la capacidad de respuesta de la población expuesta. En este contexto, el objetivo principal del trabajo es evaluar la vulnerabilidad socioambiental en la localidad de Monte Caseros, a fin de brindar una herramienta que permita profundizar en el conocimiento de la capacidad de respuesta de la población ante eventos extremos, como es el caso de las inundaciones. A partir de datos censales de 2010, se construyó un índice sintético (Índice de Vulnerabilidad Socioambiental, IVSA) integrando 20 indicadores que contemplan 5 dimensiones de la vulnerabilidad socioambiental. Posteriormente, los valores obtenidos fueron analizados espacialmente considerando estudios antecedentes y observaciones realizadas en campo. Los valores más críticos (23 desvíos estándar por sobre la media) se presentan en el sector sur colindante al río Uruguay y en el área oeste próxima a la vía; las dimensiones que más inciden en el índice son la habitacional y la educativa. Los resultados obtenidos permiten afirmar que las áreas ambientalmente conflictivas y asociadas al crecimiento urbano se corresponden con las situaciones de máxima vulnerabilidad reflejadas por el índice. Los mapas elaborados constituyen un instrumento útil para generar estrategias de intervención en áreas críticas, que permitan revertir

1 Lic. en Diagnóstico y Gestión Ambiental; Especialista y Magister en Gestión Ambiental; Doctora en Geografía. Investigadora Asistente del CONICET con lugar de trabajo en el Instituto del Hábitat y del Ambiente (IHAM), Facultad de Arquitectura, Urbanismo y Diseño (FAUD), Universidad Nacional de Mar del Plata (UNMdP). Funes 3350 (CP 7600) Mar del Plata. Correo electrónico: laurazulaica@conicet.gov.ar 2 Lic. en Ecología y Conservación de los Recursos Naturales Renovables; Magister Sciencie en Gestión Ambiental del Desarrollo Urbano; Investigadora del Instituto del Hábitat y del Ambiente (IHAM), Facultad de Arquitectura, Urbanismo y Diseño (FAUD), Universidad Nacional de Mar del Plata (UNMdP). Funes 3350 (CP 7600) Mar del Plata. Correo electrónico: rferraro@mdp.edu.ar 
problemáticas ambientales relevantes, asociadas con la escasa implementación de instrumentos de gestión urbana, como es el ordenamiento territorial.

Palabras clave: problemas ambientales, inundaciones, crecimiento urbano, ordenamiento territorial.

\begin{abstract}
Cities are exposed to various natural phenomena and environmental problems that pose a challenge for local management, whose uncertainty is growing in a context of climate change. In the town of Monte Caseros, Corrientes, the rising of the Uruguay River results in flooding events, as evidenced by the end of 2015 and early 2016. These phenomena may become more or less important according to the responsiveness of the exposed population. In this context, the main objective of this study is to assess socio-environmental vulnerability in the town of Monte Caseros, in order to provide a tool to deepen the understanding of the population's responsiveness to extreme events, such as flooding. From census data from 2010, a synthetic index was created (Socio-Environmental Vulnerability Index, SEVI) by integrating 20 indicators that include 5 dimensions of socio-environmental vulnerability. Subsequently, the obtained values were spatially analyzed considering background studies and field observations. The most critical values (2-3 standard deviations above the mean) are presented in the south sector, which is adjacent to the river Uruguay and in the western area next to the road; the dimensions that affect the index the most are housing and education. The results confirm that environmentally troubled areas and the areas associated with urban growth correspond to the situations of maximum vulnerability reflected by the index. The elaborated maps are a useful tool to generate intervention strategies in critical areas in order to revert relevant environmental issues associated with poor implementation of urban management tools, such as land use planning.
\end{abstract}

Key words: environmental problems, floods, urban growth, land use planning. 


\section{Introducción}

Las ciudades se encuentran expuestas a diversos fenómenos naturales y problemas ambientales, muchos de los cuales se han intensificado a través del tiempo como consecuencia de las acciones humanas. Estos fenómenos y problemas constituyen un desafío para la gestión local, cuya incertidumbre es cada vez mayor en un contexto de cambio climático. Las ciudades conforman el escenario en el que se manifiestan los principales efectos de los procesos que, combinados con una urbanización creciente, podrían generar nuevas problemáticas sociales y ambientales, o bien profundizar las existentes.

La población de Argentina, distribuida en 24 provincias, alcanza algo más de 40 millones de habitantes y los datos censales de 2010 estiman un porcentaje de población urbana superior al 90\%. Este valor es sumamente elevado en el contexto mundial y compartido con la mayor parte de los países vecinos, ya que Uruguay, Brasil y Chile también presentan valores de entre el $92 \%$ y el $85 \%$; Bolivia y Paraguay, por su parte, aún no superan el $67 \%$ de población urbana (PET, 2011).

Los efectos del cambio climático en Argentina (SAyDS, 2009) se manifiestan en efectos hídricos extremos, destacándose el riesgo de inundaciones (CONOSUR, 2007). La principal área de riesgo se extiende por el centro y el este del país, fundamentalmente en las zonas bajas o con escasa pendiente expuestas a las crecidas de los ríos.

Dentro de la Cuenca del Plata, los derrames de los ríos Paraná, Paraguay y Uruguay en el territorio argentino están gobernados por los aportes que se producen aguas arriba, fuera del territorio nacional, lo cual indica la relativa incidencia de los afluentes en la conformación del escurrimiento de los mismos (Paoli, 2004).

En la provincia de Corrientes, las cuencas de los ríos Paraná y Uruguay tienen un régimen pulsátil en concordancia con el ritmo climático y las alturas hidrométricas de sus niveles de base; el drenaje es altamente dependiente de las condiciones topográficas con redes desintegradas que, al aumentar los aportes pluviométricos, se integran parcialmente, no sólo en los ejes principales, sino también en los sistemas propios de los interfluvios (Serra, 2002).

La ciudad de Monte Caseros se localiza en el sector sudeste de la provincia de Corrientes, sobre la margen izquierda del río Uruguay. El sitio fundacional de la localidad es elegido por ser el único que ofrece tierras altas a la vera del río Uruguay ya que se ubica como cabecera exclusiva no inundable entre las desembocaduras de los arroyos Ceibo, al norte, y Mamangá, al sur, distantes entre sí unos 10 kilómetros; al norte de la cabecera donde se asienta la ciudad puede identificarse una cuenca menor, en la que confluyen los arroyos Santa Lucía y Muñoz (Vio, 2009). 
De acuerdo con Vio (2009), entre ambos extremos del frente fluvial se desarrolla una costa de cota de inundación variable históricamente, caracterizada antes de la construcción de la represa de Salto Grande por sus playas de arenas gruesas y canto rodado, prácticamente en la totalidad de esta extensión de 10 kilómetros; a partir de la puesta en servicio de la represa, estas playas prácticamente desaparecen en ciertas épocas del año, como efecto de la elevación de la cota del río.

Las crecientes extraordinarias del río Uruguay, aunque acíclicas, se producen cada 10 años. Los informes de la Comisión Administradora del Río Uruguay (CARU, 2016) destacan la incidencia del fenómeno de “El Niño" en las crecidas del río. Según la misma fuente, el caudal de aporte total al embalse de Salto Grande aumentó bruscamente entre $8000 \mathrm{~m}^{3} / \mathrm{seg}$ el 6 de diciembre de 2015 y $33.800 \mathrm{~m}^{3} / \mathrm{seg}$ el 26 de diciembre de ese año, por el fuerte impacto de las intensas y persistentes lluvias. Además, el informe señala que los resultados de diversos modelos de pronósticos del ENSO (EI Niño-Oscilación del Sur) muestran que las condiciones de “El Niño" se mantienen en el primer trimestre de 2016, declinando rápidamente hacia el otoño.

En Monte Caseros, las crecidas del río Uruguay se traducen en eventos de inundaciones, siendo que en 1983 se alcanzó la máxima crecida (10,19 m). Los registros de Prefectura Naval comprendidos desde 2015 y hasta abril de $2016^{3}$ indican alertas (entre 7,5 y 8,5 m) en los siguientes momentos: 16/10/2015-21/10/2015; 20/11/2015, 22/12/2015; y 04/01/2016-08/01/2016. Asimismo, dichos registros denotan condiciones de evacuación (más de 8,5 m) entre el 23/12/2015 y el 03/01/2016.

A los eventos de inundaciones en la ciudad se suman otras amenazas (contaminación de cursos de agua superficial por vertidos cloacales e industriales, exposición a agroquímicos, presencia de basurales a cielo abierto, entre otros) que ponen a la población en situación de riesgo.

Según Viand y González (2012), si bien la discusión conceptual del riesgo es amplia (Beck, 1998; Wynne, 1992; Wilches Chaux, 1993), desde el punto de vista analítico, se pueden identificar dos dimensiones constitutivas: la peligrosidad, definida como la probabilidad de ocurrencia de un fenómeno natural o tecnológico con potencialidad de generar daño (Natenzon, 1998); y la vulnerabilidad, que refiere a las condiciones sociales, económicas, culturales, etc., que exponen a una población y la hace propensa a ser afectada y sufrir daño respecto a una peligrosidad (Lavell, 1996).

Las consecuencias de fenómenos extremos como los evidenciados hacia fines de 2015 y principios de 2016 pueden adquirir mayor o menor relevancia de acuerdo con la capacidad de respuesta de la población expuesta a esta amenaza. En este contexto vale preguntarse ¿Existen diferenciaciones territoriales en los alcances y consecuencias de 
la problemática de inundaciones en el interior de Monte Caseros? El concepto de vulnerabilidad socioambiental parece apropiado para responder al menos en parte a ese interrogante.

En términos generales, la vulnerabilidad es entendida como un proceso multidimensional que confluye en el riesgo de la población a sufrir daños ante los cambios o la permanencia de condiciones negativas internas o externas. El nivel de vulnerabilidad, es decir, la capacidad que posee la población para prevenir, reducir o afrontar los riesgos, depende de la interacción de varios factores de origen natural y/o socioeconómico.

Wilches Chaux (1993) define al concepto como la incapacidad de una comunidad para absorber, mediante el autoajuste, los efectos de un determinado cambio en su entorno, es decir su inflexibilidad o incapacidad para adaptarse a ese cambio. Sánchez González y Egea Jiménez (2011) señalan que hay investigaciones que abordan las interacciones entre las cuestiones ambientales y sociodemográficas abarcando ámbitos diversos, siendo el urbano el espacio más atractivo para este tipo de investigaciones debido a que en él la vulnerabilidad se asocia con riesgos crecientes y dinámicos.

Dado que los factores que inciden en la vulnerabilidad son internos y también externos (determinados por las condiciones del ambiente), es posible hacer referencia a condiciones de vulnerabilidad socioambiental. Este tipo de vulnerabilidad, definida en estudios previos (Buch y Turcios, 2003; Ávila García, 2008; Bello Sánchez et al., 2009; Zulaica y Ferraro, 2010; Cabral y Zulaica, 2015; Daga et al., 2015), está determinada por las características intrínsecas de la población y su entorno próximo para enfrentar las dificultades o riesgos. Dichas características comprenden un conjunto de factores (sanitarios, educativos, habitacionales, económicos, sociales, entre otros), cuyo grado de debilidad define distintos niveles de vulnerabilidad socioambiental frente al problema estudiado.

Sobre la base de las conceptualizaciones anteriores, el objetivo principal de este trabajo es evaluar la vulnerabilidad socioambiental en la localidad de Monte Caseros, a fin de brindar una herramienta que permita profundizar en el conocimiento de la capacidad de respuesta de la población ante eventos extremos, como es el caso de las inundaciones, y generar estrategias para revertir las situaciones más críticas.

\section{El área de estudio}

El Área de Triple Frontera en la que se asientan las localidades de Monte Caseros (Argentina), Bella Unión (Uruguay) y Barra do Quarai (Brasil), incide en la distribución territorial de la población, definiendo un patrón de asentamiento que prioriza la zona costera del río Uruguay, principalmente por la posibilidad de intercambio de bienes y servicios con los países limítrofes. 
El área de estudio, que constituye la localidad de Monte Caseros, pertenece al Departamento que lleva el mismo nombre en la provincia de Corrientes y fue ajustada en este trabajo según la información de base disponible, es decir, considerando los radios censales de 2010 (INDEC, 2010), como se muestra en la Figura 1. En esa misma Figura se destacan los barrios principales de la localidad a los que se hará referencia en el presente artículo.

Figura 1: Área de estudio.

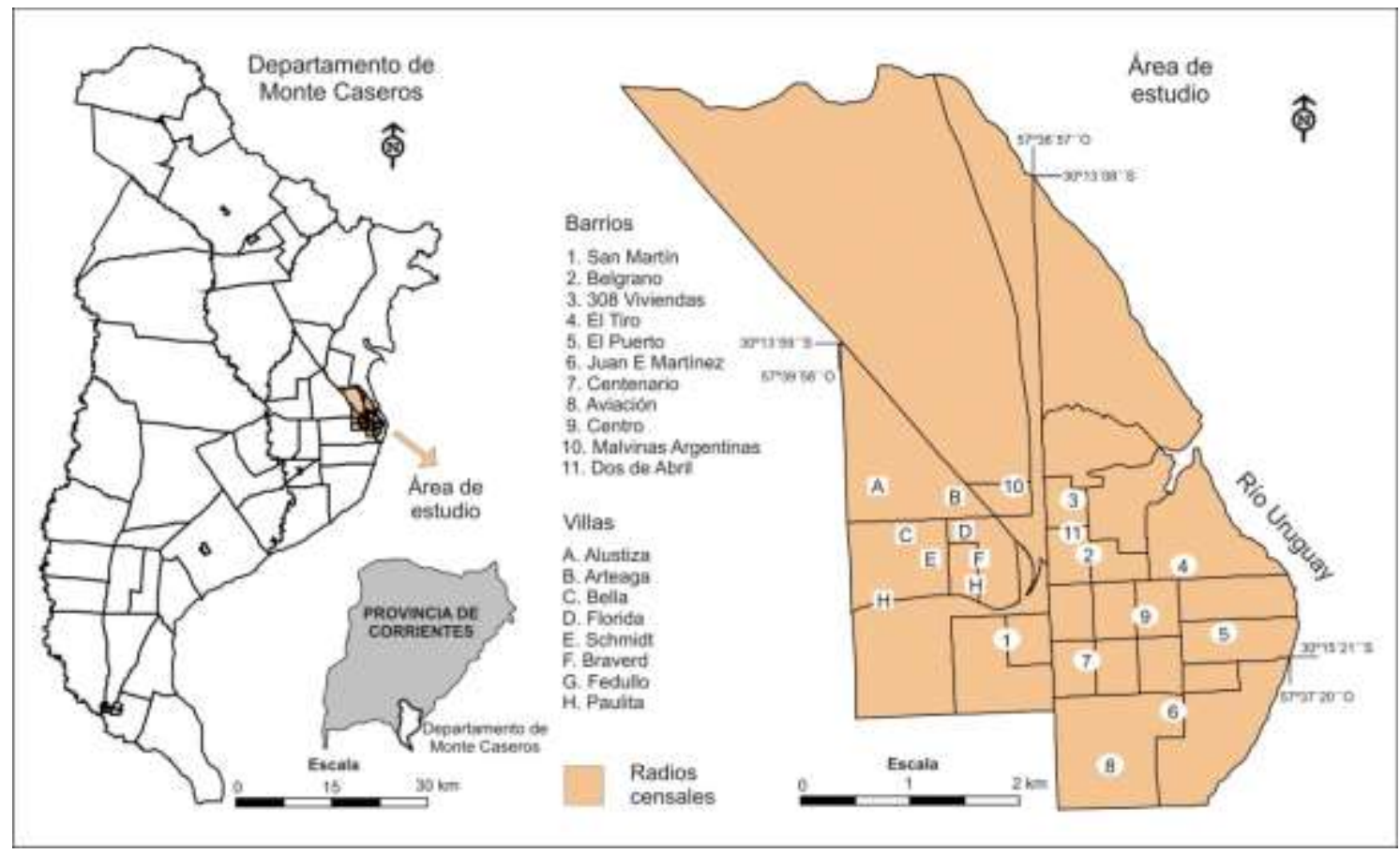

Fuente: Elaboración personal a partir de los radios censales del INDEC (2010).

La población del área de estudio alcanzó en 2010 los 23.470 habitantes que se asientan en la localidad y sus alrededores, y constituyen el 64,6\% de la población total del Departamento de Monte Caseros. En el último período intercensal (2001-2010) se verifica un crecimiento poblacional del 5,3\% en el área de estudio.

Los datos de 2010 indican que la distribución de la población por sexo muestra una mayor proporción de mujeres (12.148) que supera levemente a la de varones (11.322).

La estructura de edades permite verificar la presencia importante de población infantil, que agrupa el 41,6\% de la población total en el rango de entre 0 y 14 años. Esta composición muestra una población en crecimiento.

Según lo expresado en el Plan Estratégico de Desarrollo Productivo (Cravacuore et al., 2011), durante cien años la principal actividad económica local fue la ferroviaria, seguida por los servicios públicos, en especial aquellos vinculados con el Estado nacional. Desde comienzos de la década de los noventa, el cierre de los talleres y el achicamiento de las unidades militares acrecentaron el peso de la producción primaria en general y de la citricultura 
en particular. La citricultura ocupa el principal lugar en términos de valor bruto de producción, seguido por la ganadería, la actividad forestal y otras actividades agrícolas.

\section{Metodología}

El análisis de la vulnerabilidad socioambiental de Monte Caseros, se centró en la construcción de un índice sintético, Índice de Vulnerabilidad Socioambiental (IVSA) que permitió sintetizar distintos factores que inciden en la vulnerabilidad, intentado responder a objetivos sociales de mejorar la comunicación y establecer comparaciones (Hammond et al., 1995). De acuerdo con Schuschny y Soto (2009), se trata de un indicador compuesto, es decir una representación simplificada que busca resumir un concepto multidimensional en un índice simple (unidimensional) con base en un modelo conceptual subyacente.

En la construcción del IVSA se consideraron especialmente los aportes de Wilches Chaux (1993) y Ávila García (2008) debido a que incorporan en sus enfoques las dimensiones sociales inherentes a la problemática. El Índice se elabora contemplando distintas dimensiones implicadas en el concepto. En este caso se incluyeron las siguientes dimensiones de la vulnerabilidad socioambiental: sanitaria, educativa, habitacional, económica y social. En sus definiciones, se partió de estudios antecedentes en los cuales se construyeron índices de vulnerabilidad (Zulaica y Ferraro, 2010; Cabral y Zulaica, 2015; Daga et al., 2015), ajustando las variables e indicadores al objetivo de este trabajo. Con et al. (2011) señalan que la mayoría de los antecedentes metodológicos remiten a la construcción de índices de vulnerabilidad, utilizando como fuente encuestas y datos censales. En este caso, la selección de indicadores corresponde a datos censales de 2010 (INDEC, 2010) y, por lo tanto, las unidades de referencia espacial fueron los radios censales de ese año. Los datos se procesaron con REDATAM + SP.

Se seleccionaron 20 indicadores que se agruparon en las 5 dimensiones. Como se trata de un índice de vulnerabilidad, el mismo expresa las condiciones más críticas de cada indicador; esta es la razón por la que se utilizan indicadores de "costo", que expresan situaciones negativas en el contexto analizado. Luego, los valores obtenidos para cada uno de los indicadores seleccionados se estandarizaron con la finalidad de transformarlos en unidades adimensionales que permitan establecer comparaciones (Buzai y Baxendale, 2002; Buzai, 2003). En este caso, se utilizó la técnica de Puntaje Z. Este procedimiento obtiene el puntaje estándar calculando la sumatoria, el promedio y el desvío estándar de cada indicador. Para el cálculo se utilizó la siguiente fórmula:

$z=\frac{x i-x}{\sigma}$

Donde: $x_{i}=$ el dato original a ser estandarizado, $x=$ promedio de los valores del indicador, $\sigma=$ desvío estándar de los valores del indicador. 
Posteriormente, para cada dimensión se construyó una Matriz de Correlaciones, la cual contiene los resultados obtenidos de la aplicación del coeficiente de correlación $r$ (de Pearson) entre los distintos indicadores. Este procedimiento se aplicó para detectar fuertes asociaciones entre los indicadores y distinguir aquellos que podrían ser redundantes (Pérez Morales et al., 2016). De esta manera se identificaron las máximas correlaciones y se obtuvieron los “pares recíprocos” en cada dimensión, que definen macroindicadores.

Las dimensiones y los indicadores se ponderaron respecto de un puntaje total de 1. Para cada dimensión se definió un peso uniforme de 0,2 y las ponderaciones de los indicadores y macroindicadores se distribuyeron de manera uniforme. En el caso de los macroindicadores, el valor estandarizado se definió a partir del promedio de los puntajes estandarizados de los dos indicadores contenidos en el par recíproco. En la Tabla 1 se presentan las dimensiones consideradas, indicadores-macroindicadores y ponderaciones.

EI IVSA se calculó a partir de la sumatoria de los valores estandarizados y ponderados de cada indicador. Los resultados obtenidos para el índice y cada una de las dimensiones se representaron espacialmente utilizando un Sistema de Información Geográfica (QGIS). Posteriormente, los valores obtenidos fueron analizados espacialmente, considerando estudios antecedentes y observaciones realizadas en campo.

Tabla 1: Dimensiones consideradas en la construcción del IVSA, indicadores-macroindicadores y los valores de ponderación (VP).

\begin{tabular}{|c|c|c|}
\hline Dimensión & Indicadores - Macroindicadores & VP \\
\hline \multirow{2}{*}{ Educativa } & $\begin{array}{l}\% \text { de población que no sabe leer ni escribir - \% de población que nunca asistió a } \\
\text { un establecimiento educativo }\end{array}$ & 0,1 \\
\hline & $\begin{array}{l}\% \text { de población que no finalizó estudios secundarios - \% de población que no usa } \\
\text { computadora }\end{array}$ & 0,1 \\
\hline \multirow{5}{*}{ Sanitaria } & $\begin{array}{l}\% \text { de hogares que no tienen botón o cadena en el inodoro - \% de hogares sin } \\
\text { heladera }\end{array}$ & 0,04 \\
\hline & $\%$ de hogares sin baño & 0,04 \\
\hline & \% de hogares con calidad de conexión a los servicios básicos insuficiente & 0,04 \\
\hline & $\%$ de hogares que no utilizan agua de red para beber o cocinar & 0,04 \\
\hline & $\%$ de hogares sin cobertura de red cloacal & 0,04 \\
\hline \multirow{4}{*}{ Habitacional } & $\begin{array}{l}\% \text { de hogares con Calidad de materiales de la vivienda IV - \% de hogares con } \\
\text { calidad constructiva insuficiente }\end{array}$ & 0,05 \\
\hline & $\%$ de hogares en viviendas de tipo inconveniente & 0,05 \\
\hline & $\%$ de hogares con hacinamiento critico & 0,05 \\
\hline & $\%$ de hogares en viviendas y terrenos que no son propios & 0,05 \\
\hline \multirow[t]{2}{*}{ Social } & $\begin{array}{l}\% \text { de población de } 0 \text { a } 4 \text { años de edad - \% de población con Necesidades Básicas } \\
\text { Insatisfechas }\end{array}$ & 0,1 \\
\hline & Densidad poblacional & 0,1 \\
\hline \multirow{2}{*}{ Económica } & Índice de Dependencia Potencial & 0,1 \\
\hline & \% de población desocupada & 0,1 \\
\hline
\end{tabular}

Fuente: Elaboración personal a partir de datos censales del INDEC (2010). 


\section{Resultados}

Siguiendo el procedimiento expresado en la metodología, en la primera parte, se analizan los resultados obtenidos para cada una de las dimensiones que componen el IVSA, luego su distribución espacial y, finalmente, se establecen las relaciones más significativas entre los sectores con mayor vulnerabilidad, áreas actual o potencialmente inundables y el crecimiento urbano en la localidad.

\section{Dimensiones de la vulnerabilidad socioambiental}

En primer lugar, se considera importante realizar un análisis de las distintas dimensiones que integran el IVSA a fin de profundizar en el conocimiento de la distribución de los indicadores que abordan aspectos específicos de la vulnerabilidad socioambiental. La Figura 2 muestra la distribución espacial de los resultados obtenidos para las cinco dimensiones que componen el índice. 
Figura 2: Dimensiones que componen el IVSA.

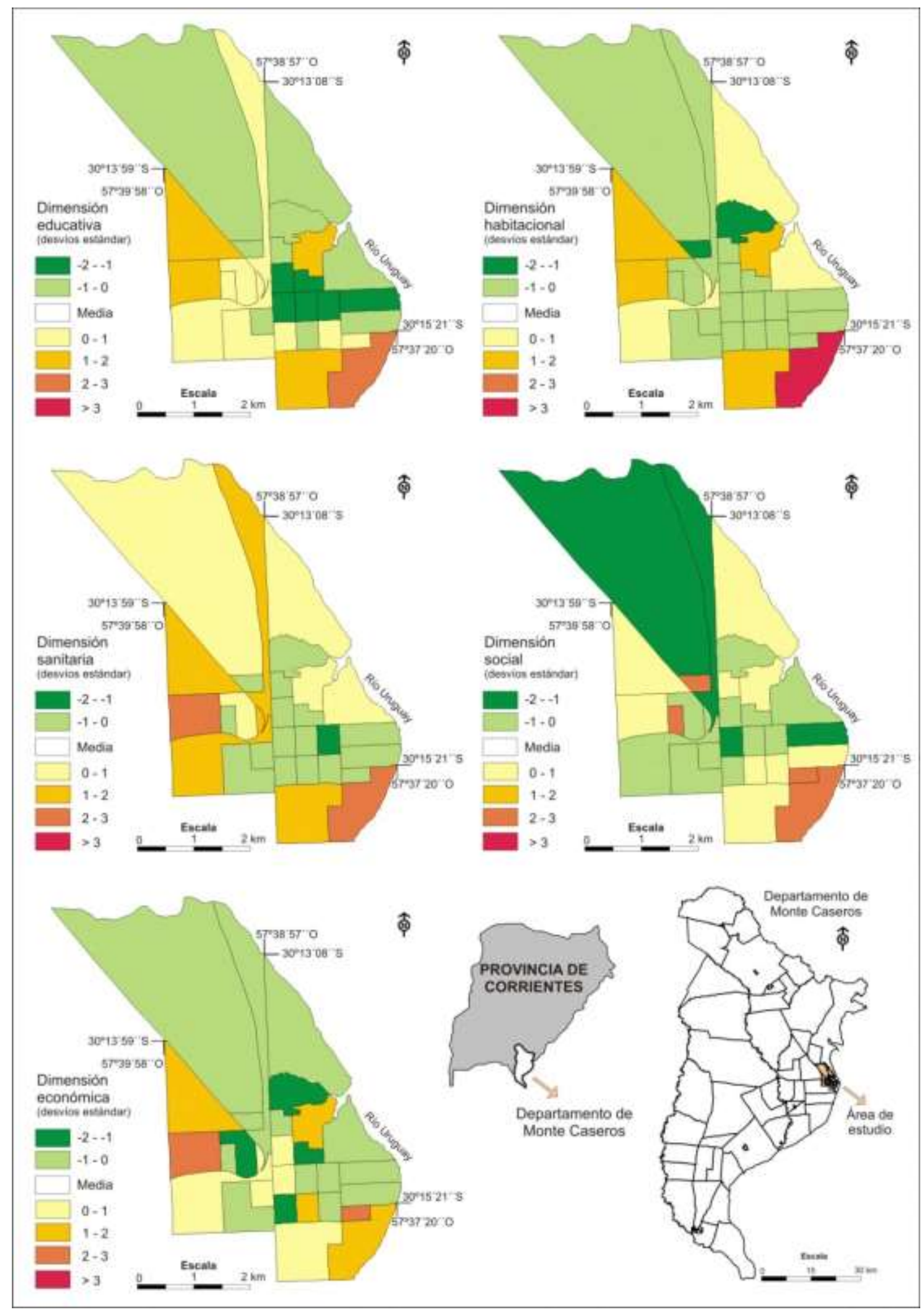

Fuente: Elaboración personal a partir de datos censales del INDEC (2010). 


\section{Dimensión educativa}

La dimensión educativa integra dos macroindicadores. El primero de ellos se corresponde con el porcentaje de población analfabeta y con aquella mayor de 3 años que nunca asistió a un establecimiento educativo.

El último censo nacional registró en el área de estudio un 7,8\% de población que no sabe leer ni escribir, localizándose fundamentalmente en los sectores colindantes con el río Uruguay y en aquellos más alejados del área urbana.

La condición de asistencia refiere a la población que asiste actualmente, asistió en el pasado o nunca ha asistido a un establecimiento educativo. Cuando se analizan los datos del área de estudio en función de este aspecto considerando sólo la población de 3 años y más, se verifica que el 59,7\% asistió a un establecimiento educativo, el $31,2 \%$ asiste y apenas el 3,7\% nunca asistió. Cuando se comparan los porcentajes de población que nunca asistió a un establecimiento educativo al interior del área de estudio, se observan los valores más favorables en el área central de la localidad, en tanto que los más críticos se presentan en las áreas periféricas, incluyendo los barrios El Tiro, Juan E. Martínez, Aviación y las villas Alustiza, Arteaga, Bella Florida, Schmidt, Bravard, Fedullo y Paulita.

El otro "par recíproco" de esta dimensión se corresponde con el porcentaje de población que no posee secundario y con el porcentaje de población en hogares que no usan computadora.

El 29,3\% de la población del área posee nivel secundario. Los datos publicados por Cravacuore et al. (2011) indican que aún una parte importante de la población cuenta con un limitado nivel educativo que condiciona fuertemente el acceso al mercado laboral.

Por otra parte, el Censo 2010 incorporó una pregunta que indaga sobre la capacidad de utilización de las nuevas Tecnologías de Información y Comunicación (TICS) de la población. La inclusión digital es una temática clave en la actualidad, dada la importancia fundamental de las nuevas tecnologías en lo referido a la inserción laboral y social de cada individuo (INDEC, 2012). En ese sentido, la indagación sobre el uso de computadora constituye una aproximación a la alfabetización digital y es de suma utilidad para conocer el nivel de utilización de las tecnologías de la información y la comunicación por parte de la población.

Un dato apropiado para evaluar este acceso es analizar la población de 3 años y más que usa computadora. En el área en su conjunto, el 46,6\% de la población usa computadora. Las situaciones más críticas se presentan en el sector sur de la localidad y en un área de Villa Paulita. 


\section{Dimensión habitacional}

En el caso de la dimensión habitacional, se tomaron cinco indicadores provistos por el Censo, dos de los cuales se agruparon en un macroindicador.

Una parte importante de las condiciones habitacionales se vinculan directamente con características de las viviendas. Así, se toma en este análisis el indicador hogares que habitan en una vivienda de tipo inconveniente; esto incluye, según las definiciones censales, pieza de inquilinato, pieza de hotel o pensión, casilla, local no construido para habitación o vivienda móvil, excluyendo casa, departamento y rancho.

Los datos del censo de 2010 para el área de estudio muestran que las viviendas de tipo inconveniente apenas alcanzan el 3,5\%. Las condiciones más desfavorables para este indicador se alcanzan en el sector oeste del área de estudio, que se corresponde con Villa Alustiza, Villa Arteaga, Villa Florida, Villa Schmidt y Fedullo.

En cuanto a las viviendas, el anteúltimo censo (INDEC, 2001) consideró en el relevamiento el indicador denominado "calidad de los materiales de la vivienda", con el fin de determinar las características edilicias de las viviendas. La clasificación llamada CALMAT (calidad de los materiales) resume en cuatro categorías las condiciones de los pisos, paredes, techos, elementos de aislación y terminación. En este caso se consideró la Categoría IV, la cual caracteriza a las viviendas que presentan materiales no resistentes ni sólidos o de desecho al menos en uno de los parámetros.

El 3,1\% de las viviendas del área integraban en 2010 la última categoría. La situación más desfavorable, se presenta en el sector sur colindante al río Uruguay (barrio Juan E. Martínez) y hacia el oeste en Villa Alustiza, Villa Arteaga, Villa Florida, Villa Schmidt y Fedullo.

La calidad constructiva de la vivienda es otro indicador interesante que se obtiene a partir de la calidad de los materiales con los que está construida la vivienda y las instalaciones internas de servicios básicos (agua de red y desagüe) de las que dispone. Considerando los aspectos mencionados, se definen tres categorías. En este caso se considera la calidad insuficiente. Al analizar la peor condición en el área de estudio, se observa que el 8,9\% de las viviendas presentan calidad constructiva insuficiente. La distribución porcentual en las distintas unidades de análisis permite destacar la situación más crítica en el sector sur (barrios Juan E. Martínez y Aviación) y al oeste (Villa Bella, Villa Schmidt y Villa Fedullo).

El hacinamiento se considera crítico cuando se contabilizan más de tres personas por cuarto. En el área estudiada, el 4,0\% de los hogares se encuentra en esta situación. La unidad que reviste la peor condición de este indicador (19,0\%) es el sector sudeste, que incluye el barrio Juan E. Martínez. 
El 68,1\% de las viviendas y terrenos del área se encuentran habitados por sus propios dueños. En este caso, las situaciones más críticas corresponden a zonas colindantes con el río Uruguay situadas al norte y sur. En estas últimas se incluyen los barrios Juan E. Martínez y Aviación.

\section{Dimensión sanitaria}

En cuanto a la dimensión sanitaria, se dispone de dos datos interesantes que aseguran ciertas condiciones de salubridad en los hogares: tenencia de botón o cadena en el inodoro y la disponibilidad de heladera. Este último es de vital importancia para garantizar la seguridad alimentaria de la población. La conservación apropiada de los alimentos evita enfermedades; por lo tanto, la heladera es considerada un artefacto necesario para garantizar la calidad de vida de las personas (INDEC, 2012). Ambos indicadores componen el único macroindicador que integra esta dimensión.

Cuando se analiza el indicador tenencia de botón o cadena en el inodoro se observa que el 92,1\% de los hogares cuenta con alguno de estos elementos. La situación más desfavorable en el área de estudio corresponde al sector sur de la localidad, barrios Juan E. Martínez y Aviación, y hacia el oeste en Villa Bella y Villa Schmidt. El 93,3\% de los hogares en el área de referencia poseen heladera. Las condiciones más críticas respecto de este indicador se presentan también, como en el caso anterior, en el sector sur de la localidad y hacia el oeste.

Respecto del indicador porcentaje de hogares sin baño, un 2,4\% de los hogares del área de estudio se encuentran en esta condición. Las situaciones más críticas (10\%) se presentan en el área que bordea el río Uruguay hacia el sur, que se corresponde con el barrio Juan E. Martínez.

Los servicios de agua corriente y cloacas son brindados por la empresa privada Aguas de Corrientes S.A. Sin embargo, Cravacuore et al. (2011) mencionan que existen ciertos problemas recurrentes con la calidad del agua, la que es abastecida directamente desde el río Uruguay.

En la totalidad del área delimitada, los datos censales permiten afirmar que el 99,3\% de los hogares utilizan agua de red pública para beber o cocinar. La situación muestra diferencias al interior del área, con los valores más bajos de cobertura en el centro y norte de la localidad. En cuanto a la cobertura de red cloacal, los datos indican que la provisión en el área abastece al 91,2\% de los hogares. En el área de estudio, las situaciones más críticas (69,0\% a $77,0 \%)$ se presentan en las inmediaciones del río Uruguay hacia el norte y hacia el sur y en el oeste, en Villa Alustiza, Villa Arteaga, Villa Florida, Villa Schmidt y Villa Fedullo.

Otro indicador importante en esta dimensión tiene que ver con la calidad de conexión a servicios básicos, que refiere al tipo de instalaciones con que cuentan las viviendas para su saneamiento. Para este indicador se utilizan las variables procedencia del agua y tipo de desagüe. En este caso, se consideran las viviendas de calidad insuficiente a 
servicios. En el área, se verifica que el 5,8\% de las viviendas se incluyen en esta categoría. Los valores extremos más desfavorables se presentan también hacia el sur y el oeste.

\section{Dimensión social}

El macroindicador incluido en esta dimensión involucra el porcentaje de población infantil (menor a 4 años) que es la más susceptible ante las amenazas y el porcentaje de población con Necesidades Básicas Insatisfechas (NBI). La población de menos de 4 años de edad alcanza el 9,0\% en el área de estudio y representa un máximo de 14,5\% en el radio situado al sur colindante con el río Uruguay y mínimos comprendidos entre $5 \%$ y $7 \%$ que se presentan fundamentalmente en las áreas centrales.

Las NBI permiten identificar a la población o a los hogares que manifiestan importantes limitaciones en su vivienda (espacio insuficiente, estructura precaria o falta de instalaciones sanitarias), en la escolaridad de los niños o en la capacidad de generar recursos económicos. Los miembros de la población con NBI se podrían considerar "pobres estructurales", es decir que requieren una importante inversión material o esfuerzo personal para superar el estado de precariedad social.

Las NBI para el área estudiada alcanzan el 9,2\% de los hogares. Las situaciones más críticas con porcentajes más altos de población en hogares con NBI se presentan en el sector que bordea al río Uruguay situado al sur, que incluye un área del barrio Juan E. Martínez. Las condiciones más favorables se alcanzan en el área central.

El último indicador considerado en esta dimensión es la densidad, que se vincula con la cantidad de personas potencialmente expuestas por unidad de superficie. El sector centro sur de la ciudad concentra la mayor cantidad de habitantes (1913) en el barrio Aviación, en tanto que las densidades más altas (33,67 hab./ha - 90,6 hab./ha) se registran en los barrios Juan E. Martínez, Malvinas Argentinas, 2 de Abril y zonas de Villa Bravard y Villa Paulita.

\section{Dimensión económica}

Esta dimensión incluye dos indicadores: Índice de Dependencia Potencial (IDP) y porcentaje de población desocupada.

Como indicador de dependencia potencial, el último censo desarrolla el IDP, el cual brinda una idea aproximada de la carga de dependencia económica de una población, ya que pone en relación a los grupos de población "potencialmente inactivos" respecto de la "población potencialmente activa". El IDP para el área en su conjunto es 0,6, alcanzando un máximo de 0,78 en sector sur, próximo al río Uruguay. Más allá de los resultados obtenidos, es importante destacar que esta dependencia es potencial y responde sólo a la carga demográfica, debido a que la estimación de la dependencia efectiva requeriría incluir en el denominador a los efectivamente activos (entre 
los que pueden encontrarse personas en edad pasiva que trabajan y personas en edad activa que no trabajan) y excluir del numerador a las personas en edad pasiva que integran la población económicamente activa.

El análisis de informaciones relativas a la población de 14 o más del área, en función de su condición de actividad económica, indican que un $51,3 \%$ de los habitantes del área de estudio se encontraban ocupados, un 3,1\% desocupados y el porcentaje restante corresponde a otras condiciones (jubilados o pensionados, estudiantes, otras situaciones). Cuando se analiza la población desocupada por unidades espaciales, se observa que la peor condición se alcanza en los barrios Centenario, Juan E. Martínez y Villa Bella, Villa Schmidt y Villa Fedullo.

\section{Índice de Vulnerabilidad Socioambiental}

En análisis del IVSA (Figura 3) refiere a la capacidad de la población para enfrentar situaciones de riesgo ambiental como es el caso de las inundaciones. Los valores más críticos (2-3 desvíos estándar por sobre la media) se presentan en el sector sur colindante al río Uruguay y en el área oeste próxima a la vía, que corresponde a los las villas Bella, Schmidt y Paulita.

Asimismo, en condición desfavorable de vulnerabilidad (1-2 desvíos estándar por sobre la media) se encuentran los radios colindantes a los mencionados anteriormente. En el sector centro sur, se destacan los barrios de Juan E. Martínez y Aviación, en tanto que en la zona situada al noroeste, incluye las villas Aluztiza y Arteaga.

La situación más favorable del índice (1-2 desvíos estándar por debajo de la media) se presenta en el área central, en el barrio denominado Centro y en el radio situado más al norte (308 viviendas). En condición también favorable (0-1 desvío estándar por debajo de la media) se presenta la mayor parte del área central y los radios situados hacia el norte.

Los radios que representan las condiciones más críticas de vulnerabilidad (1-3 desvíos estándar por sobre la media) agrupan 5308 habitantes, es decir un 22,6\% del total. 
Figura 3: Índice de Vulnerabilidad Socioambiental.

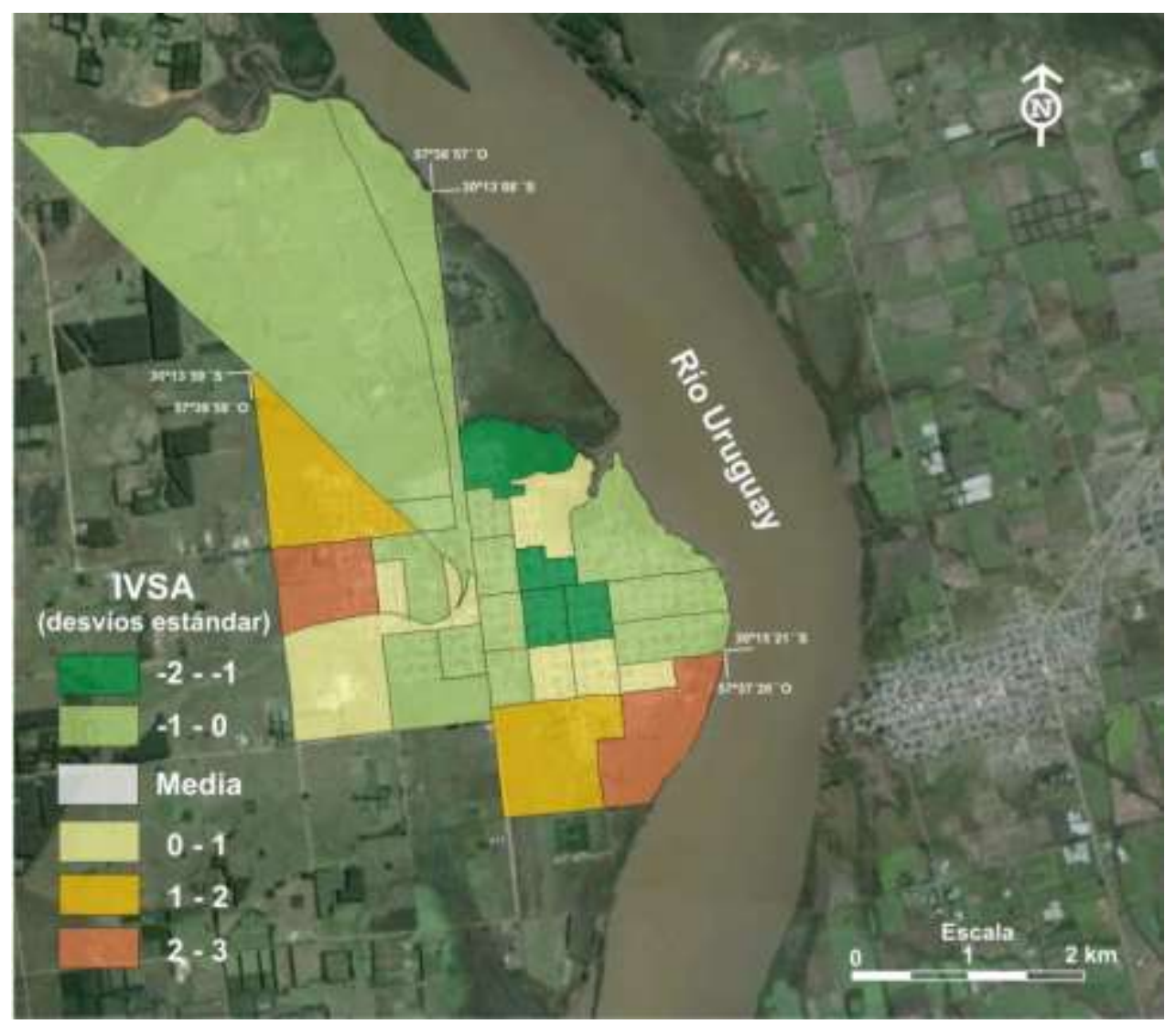

Fuente: Elaboración personal a partir de los radios censales del INDEC (2010), sobre imagen obtenida de Google Earth.

Cuando se analizan los resultados en función de cada una de las dimensiones, se verifica que la habitacional y la educativa determinan las situaciones más favorables y desfavorables en la distribución del IVSA.

Ello se corrobora al aplicar el coeficiente de correlación lineal, o $r$ de Pearson, que alcanza 0,904 para el caso de la dimensión educativa y 0,932 para la dimensión habitacional. El coeficiente mencionado, que mide el grado de relación lineal entre las variables, muestra en ambos casos una correlación positiva alta que se verifica en los gráficos de dispersión (Figuras 4a y 4b). El incremento en los valores estandarizados de las dimensiones educativa y habitacional se traduce en un incremento de 2,907 y 3,265 veces en el IVSA, respectivamente, que definen la pendiente en cada una de las rectas de regresión.

El análisis de las dimensiones habitacional y educativa revela que las situaciones más críticas se presentan en el sudeste, oeste y en un radio situado al norte de la localidad. Asimismo, resta mencionar que, en términos generales, los valores obtenidos para cada uno de los indicadores seleccionados manifiestan situaciones más favorables que cuando se los compara con el total departamental. 
Figuras 4a y 4b. Gráficos de dispersión entre los valores del IVSA y las dimensiones educativas y habitacional.

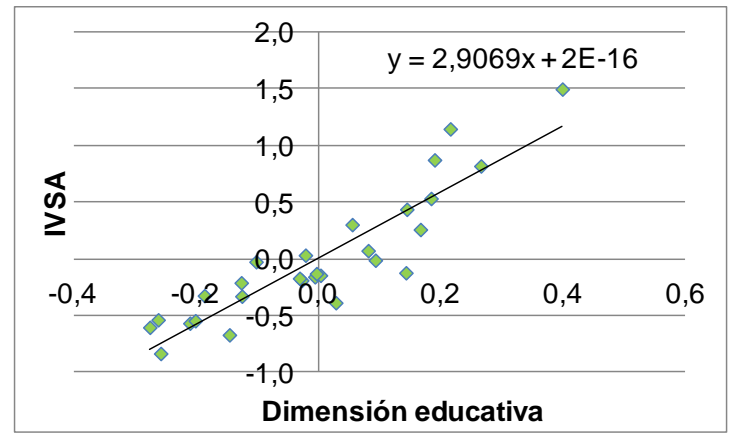

4a. Dimensión educativa.

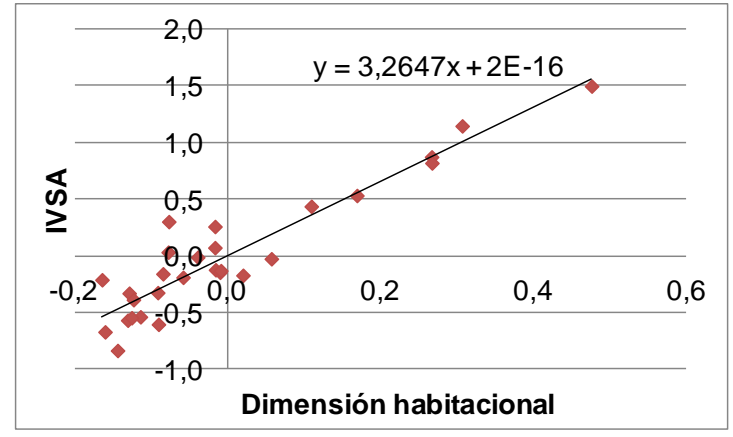

4b. Dimensión habitacional.

Fuente: Elaboración personal.

\section{Áreas inundables, crecimiento urbano y vulnerabilidad}

Como fue adelantado al principio, existe una relación estrecha entre la estructura urbana de Monte Caseros y el medio natural. Esta relación se pone en evidencia al considerar que el sitio fundacional de Monte Caseros es elegido por ser el único que ofrece tierras altas a la vera del río Uruguay.

Desde el punto de vista físico, estas favorables condiciones originarias se erigen al mismo tiempo como limitantes al crecimiento urbano hacia tres de los rumbos posibles. Así, la franja inundable frente al río Uruguay la limita hacia el este; la cuenca Santa Lucía-Muñoz, al norte; y las áreas bajas cercanas al actual Cementerio Municipal, al sur (Vio, 2009).

Esta situación constituye un problema si se considera que el crecimiento urbano en las áreas bajas compromete a la población por exposición a fenómenos ambientales. Al extenderse la localidad sobre zonas del valle de inundación del río Uruguay, son constantes los avances hídricos en función de las precipitaciones o bien, como consecuencia de la apertura de las compuertas de la represa Salto Grande. Para hacer frente a esta problemática, la ciudad posee murallones de defensa en la zona de costanera consolidada y terraplenes hacia el norte.

En Monte Caseros, el crecimiento urbano está favorecido por urbanizaciones de vivienda social, agrupadas en pequeños conjuntos en los bordes del ejido urbano. La ausencia de una planificación integral que considere las limitaciones del medio físico y también construido se traduce en problemas ambientales a los que debe dar respuesta desde la gestión local. El análisis de los conflictos urbanos realizados por Vio (2009) destaca entre otras cuestiones que:

- El conjunto habitacional situado al norte de la ciudad en áreas inundables debió ser protegido con obras de defensa que evitan la exposición de la zona a las crecidas del Arroyo Santa Lucía. 
- El área situada al sudoeste se encuentra junto al Arroyo Mamangá en la intersección con la calle Juan Pujol, constituyendo una zona "marginal" expuesta a amenazas.

- Los conjuntos habitacionales situados al sur, cercanos al cementerio, se encuentran próximos al área del basural y a las piletas de decantación de efluentes cloacales, manifestando condiciones de degradación ambiental.

Entre otras amenazas, estos sectores están expuestos en mayor o menor medida a las crecidas de los ríos cuyas consecuencias podrían intensificarse en un contexto de cambio climático. El problema no es menor si se tiene en cuenta el análisis de vulnerabilidad realizado a partir de la construcción del índice.

La Figura 5 muestra las zonas bajas y áreas inundables mencionadas anteriormente, que presentan conflictos asociados con el crecimiento no planificado de la localidad. Como se desprende del análisis, las áreas ambientalmente conflictivas y asociadas al crecimiento urbano se corresponden con situaciones de máxima vulnerabilidad reflejadas por el IVSA.

Figura 5. Áreas de crecimiento urbano no planificado.

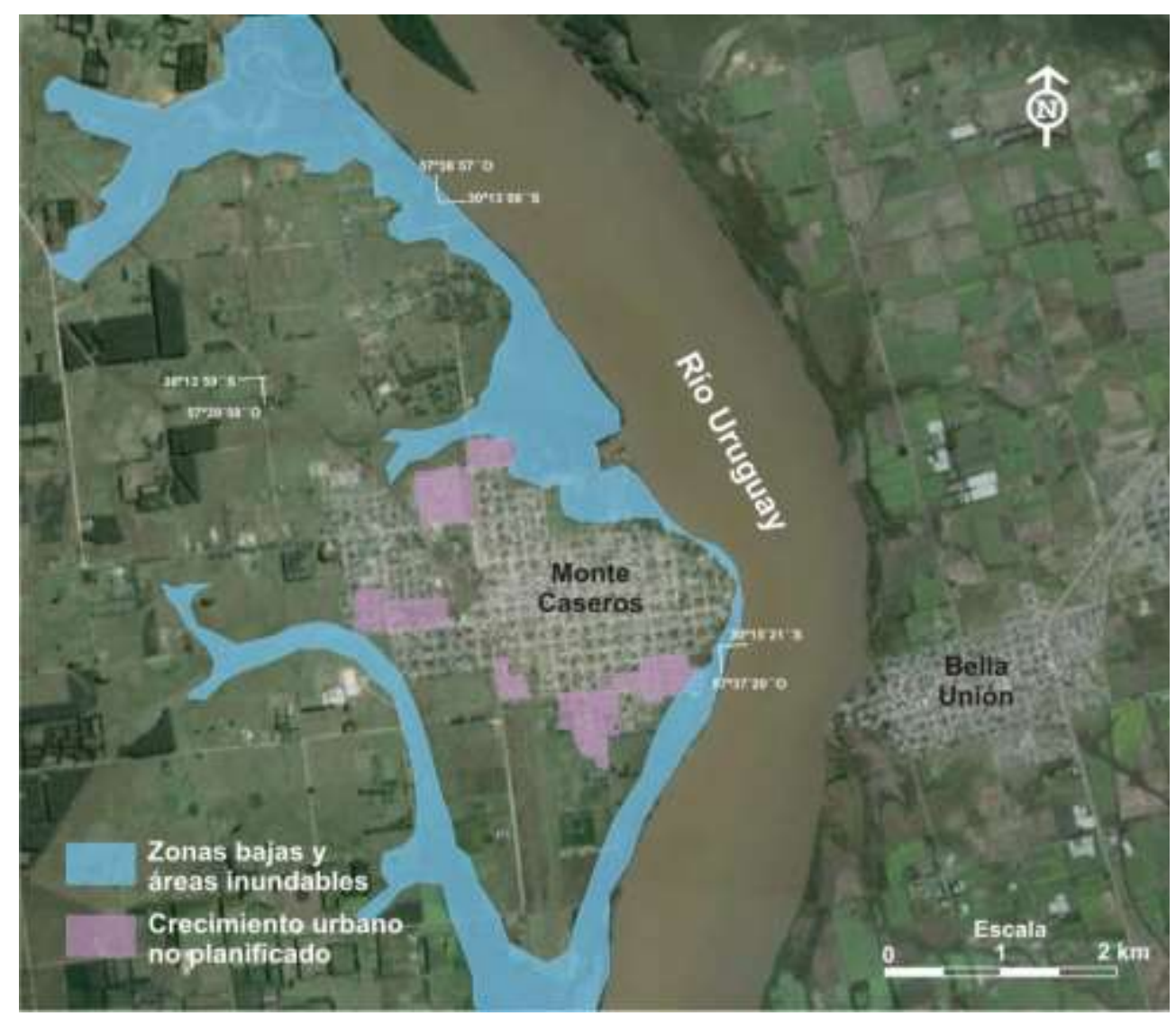

Fuente: Elaboración personal a partir de Vio (2009), sobre imagen obtenida de Google Earth. 


\section{Consideraciones finales}

La evaluación de la vulnerabilidad socio-ambiental realizada en Monte Caseros revela situaciones críticas en los sectores sudeste y oeste de la localidad. Algunos de los barrios ubicados en esas zonas se asientan en áreas bajas o inundables o bien próximos a ellas en sectores ambientalmente degradados. Así como sugieren estudios antecedentes realizados en otras áreas, el análisis de la distribución de IVSA permite establecer que sectores considerables de Monte Caseros se alejan de los logros de equidad y bienestar socioambiental acordes con los principios de sustentabilidad.

La distribución espacial del índice revela que existen correspondencias entre los resultados obtenidos para los valores extremos de los indicadores de cada una de las dimensiones consideradas en las distintas unidades espaciales. A su vez, las dimensiones que más inciden en el índice son la habitacional y la educativa. La habitacional alude fundamentalmente a las características y condiciones de la vivienda para ofrecer resistencia a eventos extremos. Está sumamente ligada a la dimensión económica, es decir, condiciones de ocupación, insuficiencia de ingresos e inestabilidad laboral que podrían explicar la dificultad o imposibilidad de recuperación ante los daños ocasionados por un fenómeno como las inundaciones. La dimensión educativa refiere fundamentalmente a la forma de acceso al conocimiento para poder enfrentar los riesgos.

Como es sabido, los elementos implícitos en estas dimensiones exceden el ámbito local; sin embargo, es posible pensar en estrategias que mitiguen las debilidades definidas a partir de los indicadores utilizados en la construcción del índice.

Aunque se considera importante profundizar el análisis a partir de la incorporación de nuevos indicadores, los aportes realizados en este estudio podrían convertirse en una herramienta factible de aplicar para establecer los distintos factores que contribuyen al aumento de la vulnerabilidad. De esta manera, se facilita el direccionamiento de los esfuerzos para tomar las medidas necesarias que permitan mitigar los fenómenos naturales y antrópicos a los que está expuesta la población de la localidad. Así, los resultados obtenidos aportan una base diagnóstica central para generar estrategias de actuación ante fenómenos ambientales, cuyos efectos se potencian en las áreas de mayor vulnerabilidad.

Además, sería interesante incorporar al análisis las percepciones sobre las dimensiones de vulnerabilidad de los habitantes de Monte Caseros, fundamentales para arribar a un diagnóstico integral de la problemática de las inundaciones. Tal como sugiere Ferrari (2012), dicha percepción constituye una parte de la realidad complementaria a las evaluaciones técnicas y es sumamente relevante para la selección y puesta en práctica de acciones para la gestión de riesgos con participación activa y continua de la comunidad afectada. 
En este sentido, los mapas elaborados constituyen un instrumento útil para generar estrategias de intervención en áreas críticas, que permitan revertir problemáticas ambientales relevantes, asociadas con la escasa implementación de instrumentos de gestión urbana, como es el ordenamiento territorial.

Por último, es importante destacar que el procedimiento metodológico empleado es factible de ser transferido a otras áreas expuestas a inundaciones y considerando un contexto de incertidumbre derivado de las consecuencias del cambio climático.

\section{Referencias bibliográficas}

ÁVILA GARCÍA, P. (2008). Vulnerabilidad socioambiental, seguridad hídrica y escenarios de crisis. Ciencias, n. 90, pp. 46-57.

BECK, U. (1998). La sociedad del riesgo. Hacia una nueva modernidad. Barcelona: Paidós.

BELLO SÁNCHEZ, W.; González Rego, R.; Cabanas Gómez, G.; Fornet Gil, P. (2009). Vulnerabilidad socio-ambiental en el centro histórico de La Habana. Cuadernos Geográficos, v. 2, n. 45, pp. 63-82.

BUCH, M. y TURCIOS, M. [comp.] (2003) Vulnerabilidad socioambiental: aplicaciones para Guatemala. En: Serie de documentos técnicos, n. 9. Guatemala: Instituto de Agricultura, Recursos Naturales y Ambiente, Universidad Rafael Landívar.

BUZAI, G. (2003). Mapas Sociales Urbanos. Buenos Aires: Lugar Editorial.

BUZAI, G. y BAXENDALE, C. (2002). La construcción regional mediante técnicas geográficas cuantitativas. Gerencia Ambiental, v. 9, n. 85, pp. 276-282.

CABRAL, V. y ZULAICA, L. (2015). Análisis de la vulnerabilidad socioambiental en áreas del periurbano de Mar del Plata (Argentina) expuestas a agroquímicos. Multiciencias, v. 15, n. 2, pp. 172-180.

CARU. (2016). Perspectivas hidrometeorológicas en el río Uruguay para el trimestre enero, febrero y marzo de 2016. Comisión Administradora del Río Uruguay. Disponible en:

http://www.caru.org.uy/web/informesprensa/INFORME\%20HIDROMETEOROLOGICO\%20EFM16.pdf

CON, M.; SUSINI, S.; CATALÁ, S.; QUINTEROS, S. (2011). Índice de Vulnerabilidad Social (IVS). Documento metodológico. Buenos Aires: Informes temáticos de la Dirección de Investigación y Estadística del Ministerio de Educación del Gobierno de la Ciudad de Buenos Aires.

CONOSUR. (2007). Impactos regionales asociados al cambio climático. Caso de estudio: Cono Sur de América del Sur. CONOSUR Sustentable.

CRAVACUORE, D.; PAULUK, M. Y ROSBACO CENTENA, V [consultores]. Plan Estratégico de Desarrollo Productivo, Monte Caseros, Provincia de Corrientes. Universidad Nacional de Tres de Febrero.

DAGA, D.; ZULAICA, L. Y VAZQUEZ, P. (2015). Evaluación de la vulnerabilidad socio-ambiental del periurbano de Mar del Plata. Estudios Socioterritoriales, n. 18, pp. 45-59. 
FERRARI, M. P. (2012). Análisis de vulnerabilidad y percepción social de las inundaciones en la ciudad de Trelew, Argentina. Cuadernos de Geografía, v. 21, n. 2, pp. 99-116.

HAMMOND, A.; ADRIAANSE, A.; RODENBURG, E.; BRYANT, D. y WOODWARD, R. (1995). Environmental Indicators: A Systematic Approach to Measuring and Reporting on Environmental Policy Performance in the Context of Sustainable Development. New York: World Resources Institute.

INDEC. (2001). Censo Nacional de Población, Hogares y Viviendas. Buenos Aires: Instituto Nacional de Estadísticas y Censos.

INDEC. (2010). Censo Nacional de Población, Hogares y Viviendas. Buenos Aires: Instituto Nacional de Estadísticas y Censos.

INDEC. (2012). Censo Nacional de Población, Hogares y Viviendas 2010; Censo del Bicentenario. Resultados definitivos, Serie B № 2, Tomo 1. Buenos Aires: Instituto Nacional de Estadísticas y Censos.

LAVELL, A. (1996). Degradación Ambiental, Riesgo y Desastre Urbano. Problemas y Conceptos: Hacia la Definición de una Agenda de Investigación. En: Fernández, M. A. y Rodríguez, L. (editores.), Ciudades en riesgo. Degradación Ambiental, Riesgos urbanos y Desastres en América Latina, pp. 21-60. Perú: Red de Estudios en Prevención de desastres en América Latina.

NATENZON, C. (1998). Riesgo, vulnerabilidad e incertidumbre. Desastres por inundaciones en Argentina. En: Seminario sobre Problemas ambientales e vulnerabilidade. Abordagens integradoras para o campo da Saude Publica. Río de Janeiro: Ministerio de la Salud.

PAOLI, C. U. (2004). Inundaciones ribereñas en el tramo del Paraná medio. En: Bertoni, J. C. (organizador), Inundaciones urbanas en Argentina, pp. 75-114. Córdoba: Global Water Partnership, GWP-SAMTAC - Programa Asociado de Gestión de Crecidas. América del Sur.

PÉREZ MORALES, A.; NAVARRO HERVÁS, F. y ÁLVAREZ ROGE, Y. (2016). Propuesta metodológica para la evaluación de la vulnerabilidad social en poblaciones afectadas por el peligro de inundación: el caso de Águilas (Murcia, sureste ibérico). Documents d'Anàlisi Geogràfica, v. 62, n. 1, pp. 133-159.

PET. (2011). Plan Estratégico Territorial: Argentina Urbana, lineamientos estratégicos para una política nacional de urbanización. Buenos Aires: Ministerio de Planificación Federal, Inversión Pública y Servicios.

SÁNCHEZ GONZÁLEZ, D. Y EGEA JIMÉNEZ, C. (2011). Enfoque de vulnerabilidad social para investigar las desventajas socioambientales. Su aplicación en el estudio de los adultos mayores. En: Papeles de Población, v. 17, n. 69, pp.151185.

SAyDS (2009). El cambio climático en Argentina. Buenos Aires: Secretaría de Ambiente y Desarrollo Sustentable. SCHUSCHNY, A. у SOTO, H. (2009). Guía metodológica, diseño de indicadores compuestos de desarrollo sostenible. Santiago de Chile: CEPAL, Naciones Unidas.

SERRA, P. Y. (2002). Características geomorfológicas e hidrográficas de la provincia de Corrientes y su incidencia en asentamientos humanos. Tesis de la carrera Doctorado en Geografía. Buenos Aires: Universidad del Salvador. VIO, M. [experta a cargo] (2009). Plan Director Urbano para Monte Caseros. Consejo Federal de Inversiones. 
VIAND, J. y GONZÁLEZ, S. (2012). Crear riesgo, ocultar riesgo: gestión de inundaciones y política urbana en dos ciudades argentinas. En: 1er Encuentro de Investigadores en Formación de Recursos Hídricos. Buenos Aires: Instituto Nacional del Agua.

WILCHES CHAUX, G. (1993). La Vulnerabilidad Global. En: A. Maskrey [comp.] Los desastres no son naturales. Bogotá: Red de Estudios Sociales en Prevención de Desastres en América Latina.

WYNNE, B. (1992). Uncertainty and environmental learning. Reconceiving science and policy in the preventive paradigm. Global environmental change, v. 2, n. 2, pp. 111-127.

ZULAICA, L. y FERRARO, R. (2010). Vulnerabilidad socio-ambiental y dimensiones de la sustentabilidad en un sector del periurbano marplatense. Estudios Socioterritoriales, n. 8, pp. 197-219. 